\title{
Enhanced photosynthetic output via dichroic beam-sharing
}

\author{
Mark D. Redwood • Raveen Dhillon • \\ Rafael L. Orozco $\cdot$ Xu Zhang • David J. Binks • \\ Mark Dickinson • Lynne E. Macaskie
}

Received: 28 April 2012/Accepted: 18 July 2012/Published online: 30 August 2012

(C) The Author(s) 2012. This article is published with open access at Springerlink.com

\begin{abstract}
Microbial solar biofuels offer great promise for future sustainable food, fuels and chemicals but are limited by low productivities and a requirement for large land areas to harvest sunlight. A $71 \%$ increase in combined photosynthetic activity was achieved by illuminating both Rhodobacter sphaeroides and Arthrospira (Spirulina) platensis from a single beam of simulated sunlight, divided using a dichroic mirror. Therefore, this technique is termed 'dichroic beamsharing', in which the complementary action spectra of two different useful micro-organisms, belonging to green and purple groups, is exploited and allows a single beam of sunlight to be shared efficiently
\end{abstract}

Electronic supplementary material The online version of this article (doi:10.1007/s10529-012-1021-5) contains supplementary material, which is available to authorized users.

M. D. Redwood $(\bowtie) \cdot$ R. L. Orozco · L. E. Macaskie Unit of Functional Bionanomaterials, School of Biosciences, University of Birmingham, Edgbaston, Birmingham B15 2TT, UK

e-mail: m.d.redwood@bham.ac.uk

R. Dhillon · D. J. Binks - M. Dickinson

School of Physics and Astronomy \& Photon Science Institute, University of Manchester,

Manchester M13 9PL, UK

\section{Zhang}

State Key Laboratory of Bioreactor Engineering, East China University of Science and Technology, 130

Meilong Road, Shanghai 200237,

People's Republic of China between separate photobioreactors. Because the action spectra of these two organisms are typical of large groups, this novel method could increase the productivity of photosynthetic micro-organisms in the production of diverse commodities.

Keywords Arthrospira (Spirulina) platensis . Bioenergy · Biohydrogen · Biofuel · Dichroic beam-sharing $\cdot$ Rhodobacter sphaeroides

\section{Introduction}

Despite the concerns over fossil fuels (Kerr 2011), the adoption of biofuels is still limited, primarily by photosynthetic efficiency (PE). For crop plants, PE is just $0.5-1 \%$ (Archer and Barber 2004) which, if biofuels are to replace fossil fuels so atmospheric carbon is stabilised by 2100 , would necessitate a doubling of agricultural land area (Gurgel et al. 2008). In contrast, photosynthetic microorganisms can produce biofuels with a significantly greater PE (up to $9 \%$ e.g. Guo et al. 2011) and can be cultivated in areas where crops cannot, such as on steep slopes, contaminated land, buildings or water, and provide a range of valuable co-products (Fig. 1) (Blankenship et al. 1995; Metzger and Largeau 2005). However, these benefits are currently offset by the cost of photobioreactors and so further improvements in PE are required for microbial solar biofuels to become viable. Attempts to enhance PE biochemically have, so far, met with limited success (e.g. Work et al. 2012 and 
references therein). In this study we demonstrate a complementary approach: dichroic beam-sharing.

Oxygenic photosynthetic microorganisms (including cyanobacteria, algae and chloroplasts; usually green) and anoxygenic purple non-sulfur bacteria have complementary action spectra and so their co-cultivation has the potential to improve overall photobioreactor efficiency. A co-culture of these is not suitable because oxygen generated by the green microorganisms inhibits the purple, and a bi-layer approach (Miyamoto et al. 1987) is limited by light scattering and absorption in the upper layer reducing light penetration to the lower. However, a dichroic mirror (DCM) has a reflection spectrum, $R(\lambda)$, (simply related to the transmittance spectrum, $T(\lambda)=$ $1-R(\lambda)$ ) that can be engineered by control of its surface coating and so can be used to direct to each microorganism its favoured part of the solar spectrum (Fig. 1). In this study, we demonstrate this approach for Arthrospira (Spirulina) platensis (a foodstuff; Raoof et al. 2006) and Rhodobacter sphaeroides ZX-5 (a hydrogen producer; Redwood et al. 2012a) and show that up to $100 \%$ increase in combined PE could be achieved.

\section{Methods}

Solar simulator

Xenon lamps are commonly used as solar simulators because their emission spectrum resembles that of the sun over most of the spectrum. This match is often further improved using specially designed 'AM1.5' filters. The American Society for Testing and Materials (ASTM) define a solar simulator as 'class A' if its emission spectrum differs from the solar spectrum by no more than $25 \%$ over all of several non-equal bands. Filtered xenon lamps are class A over the visible and near-infrared region $(400-1,000 \mathrm{~nm})$ as a whole but are only class B $(<40 \%$ difference $)$ between 800 and $900 \mathrm{~nm}$ because in this region they emit several strong spectral lines absent from the solar spectrum. The poorer spectral match in this region is not significant for many applications but is relevant to the current study because this region corresponds to the most important part of the action spectrum for purple non-sulfur bacteria. Hence, to ensure a good spectral match in this region, a filtered xenon lamp (LOT Oriel P/N LSO104; $150 \mathrm{~W}$ ) was combined with a quartz tungsten halide (QTH) lamp (Oriel P/N $60000 ; 100 \mathrm{~W}$ ), the emission spectrum of which is much closer to that of sunlight in the $800-900 \mathrm{~nm}$ spectral region. The spectrum of the Xe lamp was filtered to remove wavelengths longer than $725 \mathrm{~nm}$ whilst that of the QTH lamp was filtered to remove wavelengths shorter than $725 \mathrm{~nm}$. A water-filter further improved the spectral match in the nearinfrared by mimicking the absorbance of atmospheric $\mathrm{H}_{2} \mathrm{O}$. The beams from each lamp were overlaid (Fig. 2) to produce a combined field of illumination that was a close match to the solar spectrum, including
Fig. 1 Dichroic beamsharing (conceptual illustration). A dichroic mirror divides the solar spectrum between different organisms having complementary action spectra, such as green algae and purple bacteria. Note that algal photosynthesis, while capturing $\mathrm{CO}_{2}$, is oxygenic whereas anoxygenic photosynthesis by purple bacteria is anaerobic and $\mathrm{H}_{2}$ production is strongly inhibited by $\mathrm{O}_{2}$, hence co-culture is not practical (see text)

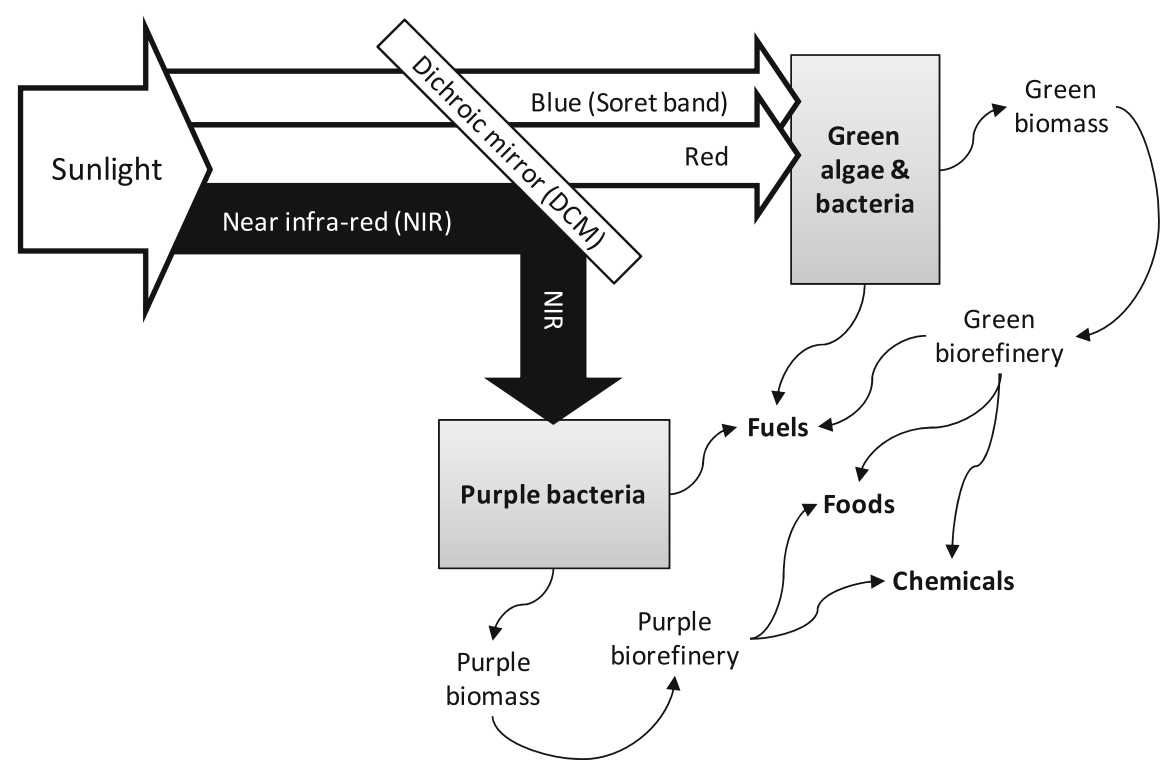




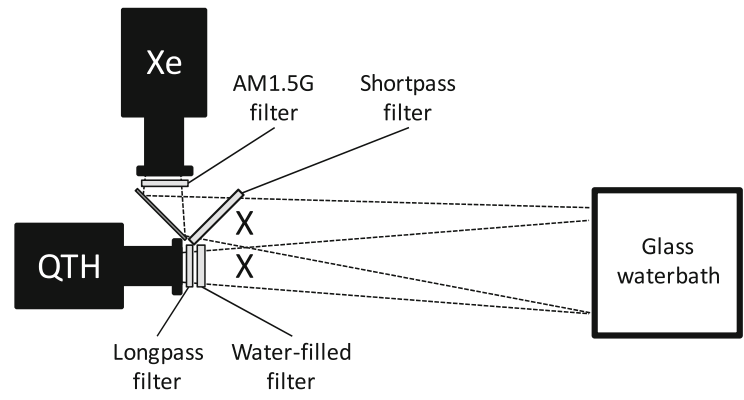

Fig. 2 Close-match solar simulator. Parts and configuration. Xe: Xenon lamp (LOT Oriel P/N LSO104; $150 \mathrm{~W}$ ). Shortpass filter: Edmund Optics (P/N NT64-668), variable cut-off: $750 \mathrm{~nm}$ at $45^{\circ}$; QTH: quartz tungsten halogen lamp (Oriel P/N 60000; $100 \mathrm{~W}$ ); longpass filter: Thorlabs P/N FGL780; water filter: glass petri dish filled with deionised water and sealed with a rubber gasket; $7 \mathrm{~mm}$ depth. The positions, when in use, of two identical dichroic mirrors (Thorlabs P/N DMLP638L) are marked by $\mathrm{X}$

in the 800-900 $\mathrm{nm}$ region important for this study. The spectra of the xenon lamp, the combined lamps and the solar spectrum were measured and compared using a spectrometer (Ocean Optics USB4000 VISNIR with Spectrasuite software in relative irradiance mode) calibrated using a blackbody source traceable to the UK National Physical Laboratory. The intensity of the combined light field could be adjusted by focusing or defocusing the beams from the lamps and was set to produce an intensity of $10 \mathrm{~W} / \mathrm{m}^{2}$, as measured by a PAR sensor (thermopile; Skye, UK, $400-1,000 \mathrm{~nm}$ ), over an area of $750 \mathrm{~cm}^{2}$. This intensity was chosen so that any light saturation effects could be neglected. Light limitation was confirmed practically; activity was proportionate to light intensity in the range $2.5-25 \mathrm{~W} / \mathrm{m}^{2}$ (see Supplementary Fig. 1). Two identical dichroic mirrors (Thorlabs $\mathrm{P} / \mathrm{N}$ DMLP638L) were used to spectrally divide the combined-lamp illumination so that the transmitted light was incident onto $A$. platensis whilst the reflected light was incident onto $R$. sphaeroides.

Strains and culture conditions

Arthrospira (Spirulina) platensis (strain no. 86.79) was purchased from Sammlung von Algenkulturen der Universität Göttingen and maintained in $400 \mathrm{ml}$ Raoof's low-cost medium (Raoof et al. 2006) at $30{ }^{\circ} \mathrm{C}$ with continuous mechanical mixing and illumination ( $9 \mathrm{~W}$ fluorescent lamp; $14 \mathrm{~W} / \mathrm{m}^{2}$ ) in a 11 water-jacketed glass vessel. $300 \mathrm{ml}$ of the culture (75\% of total) was discarded weekly and replaced with fresh Raoof's medium. Chlorophyll was measured spectrometrically according to Raoof et al. (2006) and found to be proportional to dry weight (DW) and the $\mathrm{OD}_{660}$ during the first 7 days of cultivation, after which, the chlorophyll concentration declined while DW continued to increase.

Rhodobacter sphaeroides ZX-5 was cultured as described previously (Redwood et al. 2012b), using sodium butyrate $(30 \mathrm{mM})$ as the primary carbon source. To provide consistent stocks for $\mathrm{H}_{2}$ production assays a single batch of $R$. sphaeroides cells was divided and aliquots were preserved at $-80{ }^{\circ} \mathrm{C}$. Cells were grown for $72 \mathrm{~h}\left(30^{\circ} \mathrm{C}\right.$, intermittent manual shaking) in completely filled bottles under $75 \mathrm{~W} / \mathrm{m}^{2}$ from a $300 \mathrm{~W}$ halogen lamp, harvested by centrifugation $(4,000 \times g, 15 \mathrm{~min})$ and resuspended in butyrate medium with $15 \%(\mathrm{v} / \mathrm{v})$ glycerol, before freezing in liquid $\mathrm{N}_{2}$.

Experiments used a glass waterbath $\left(30^{\circ} \mathrm{C}\right)$ receiving illumination at $10 \mathrm{~W} / \mathrm{m}^{2}$ from below by the solar simulator, in which reactors $(12 \mathrm{ml}$ total internal volume) were positioned in the beam. For A. platensis growth tests, inocula were taken from an actively growing maintenance culture, diluted to an $\mathrm{OD}_{660}$ of 0.04 (25 mg DW/1) with fresh medium, transferred $(8 \mathrm{ml})$ into reaction vials and incubated under irradiance from the solar simulator with continuous mixing by bubbling with moistened air through $18 \mathrm{G}$ needles. $\mathrm{OD}_{660}$ was recorded after 48 h. For $R$. sphaeroides $\mathrm{H}_{2}$ production tests, aliquots were thawed and diluted to $1,000 \mathrm{mg} \mathrm{DW} / 1\left(\mathrm{OD}_{660}=0.302\right)$ with fresh butyrate medium, then dispensed $(4 \mathrm{ml})$ into reaction bottles (12 $\mathrm{ml}$ internal volume), sealed with anaerobic stoppers, purged with argon (30 min) and incubated under irradiance from the solar simulator. The $\mathrm{H}_{2}$ concentration in the headspace was measured after $36 \mathrm{~h}$ as described previously (Orozco et al. 2012).

\section{Results}

Figure $3 \mathrm{a}, \mathrm{b}$ compare the action spectra of green micro-organisms and purple bacteria with the transmission and reflectance spectra of the dichroic mirror (manufacturer's data) respectively, showing a good match in both cases. Manufacturer's data were 
Fig. 3 Photosynthetic action spectra of green microorganisms (a) and purple bacteria (b) in relation to transmission and reflectance properties of a DCM, and a comparison of the spectral match of a commercial solar simulator with the one constructed for this study (c). Action spectra were compiled from ${ }^{\mathrm{a}} \mathrm{Chen}$ et al. (2010), ${ }^{\mathrm{b}}$ Haxo and Blinks (1950), ${ }^{\mathrm{c} C h e n}$ (1952), 'Nogi et al. (1985), ${ }^{\mathrm{e}}$ French (1937) and converted using Engauge Digitizer v4.1. Values from all organism-sets were pooled and trendlines were drawn by smoothing by $5 \%$ (128 points in (a) smoothed by $6 ; 36$ points in

(b) smoothed by 2).

The simulator spectra shown in (c) are relative to a solar spectrum measured at noon on a clear June day in Manchester (UK) with the same spectrometer and configuration; proximity to 1 indicates spectral match. AM1.5G:

Manufacturer's filter optimised for spectral match at ground level, $48^{\circ} \mathrm{N}$. The AM1.5 filtered xenon lamp conforms to the highest current standard for spectral match (ASTM 2010) but would be unsuitable for optical studies of purple bacteria due to the poor match in the 800-900 nm band (see text)

\section{a Action spectra of green microorganisms}
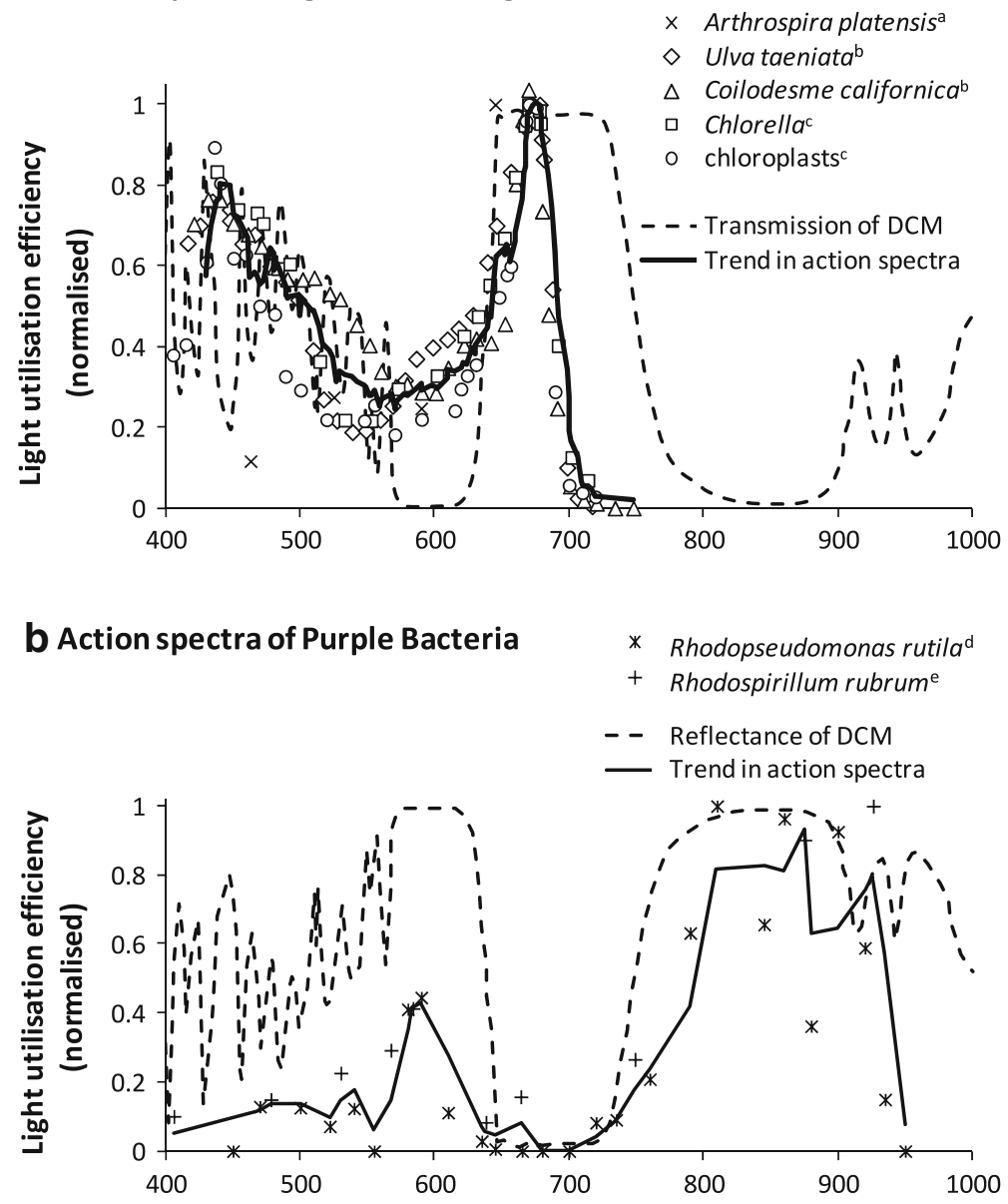

\section{Close-match solar simulation}

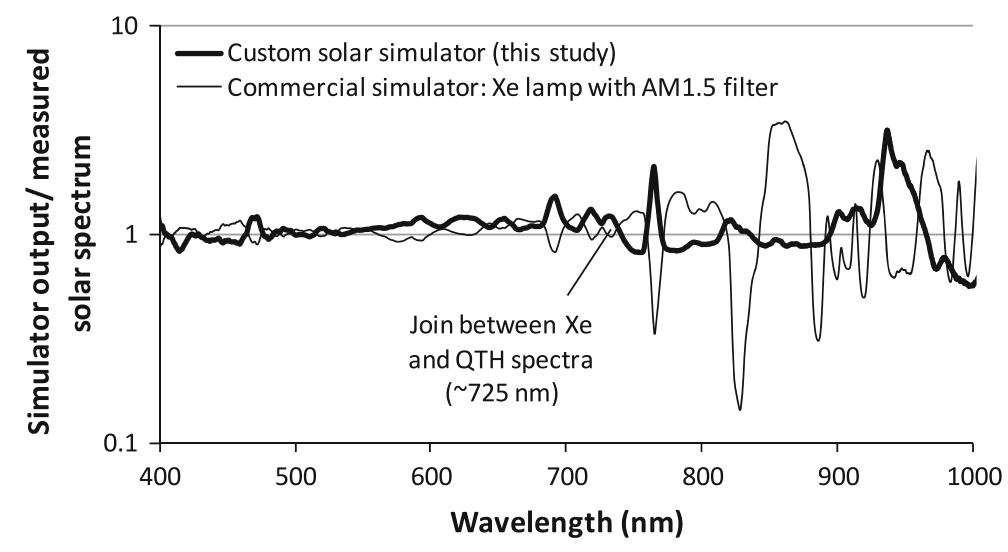

confirmed (Supplementary Fig. 2). The spectra of the filtered xenon lamp and the combined lamps relative to the solar spectrum are given in Fig. 3c, and show an improved spectral match in the 800-900 $\mathrm{nm}$ region, now class A rather than B according to ASTM classification. 

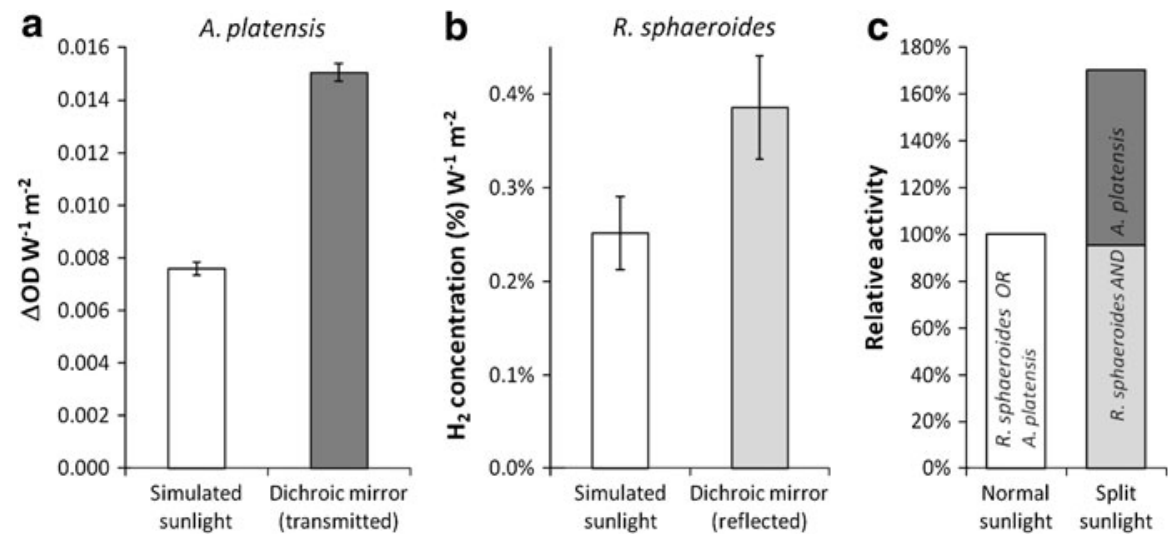

Fig. 4 Photosynthetic activity of A. platensis (a) and $R$. sphaeroides (b) and combined activity from a single beam of 'sunlight' (c). In (a) and (b), activities are expressed per unit of light intensity; the reflected and transmitted intensities were, 6.2 and $3.8 \mathrm{~W} / \mathrm{m}^{2}$, respectively. In (c), the stacked dark and pale bars represent the combined activities of $A$. platensis under transmitted sunlight (dark) and $R$. sphaeroides under reflected

Application of the dichroic mirror reduced the light supplied to A. platensis (transmitted light) by $62 \%$ but the growth only by $25 \%$. The difference was statistically significant ( $t$ test, $P<5 \%$ ). Figure 4a shows that, in relation to the total light intensity, the cultures with transmitted light were twice as efficient as those using a direct beam. Conversely, for $\mathrm{H}_{2}$ production by $R$. sphaeroides the dichroic mirror reduced the supplied light (reflected) by $38 \%$ causing no significant difference in photosynthetic activity with an average productivity equivalent to $95 \%$ of the control (full spectrum). Figure $4 \mathrm{~b}$ shows that, in relation to the total light intensity, the cultures with reflected light were 1.5 times as efficient as those using a direct beam. Figure $4 \mathrm{c}$ shows that the combined photosynthetic activity was equivalent to $170 \%$ in comparison to either reactor individually.

\section{Discussion}

The dichroic mirror provided almost complete reflection of blue and NIR wavelengths corresponding to the action maxima of purple bacteria (Fig. 3) and, accordingly, the mirror caused no detectable reduction in photosynthetic activity. Similarly, the mirror provided almost complete transmission of red wavelengths corresponding to the action maximum of green organisms. However, 50\% of light was lost through sunlight (pale), relative to either singly under complete sunlight (shown as a white bar for comparison). a Shows means and standard errors from four independent experiments with and without the dichroic mirror each including 6 replicates. b Shows four independent experiments with the mirror and three in 'sunlight', each including 4 replicates. $\triangle \mathrm{OD}$ : increase in $\mathrm{OD}_{660}$

reflection in the $400-550 \mathrm{~nm}$ band, which represents $\sim 50 \%$ of the total action for green organisms. Therefore, the overall reduction in active radiation was $\sim 25 \%$ which corresponds to the observed $25 \%$ reduction in growth for $A$. platensis. Hence, the observed $25 \%$ reduction in activity is attributed to the partial reflection of useful 'blue' $(400-550 \mathrm{~nm})$ light. Further mirror development of dichroic mirrors would aim to increase transmission in the 'blue' band, potentially increasing the combined photosynthetic activity from $170 \%$ towards the limit of $200 \%$.

The present study demonstrates the biological compatibility of dichroic beam-sharing with one pair of useful organisms. However, the action spectrum of A. platensis is typical of green micro-organisms of diverse genera and the chloroplasts of higher plants (Fig. 3), and so this technique should be applicable to other organisms with useful products. Further work is also required to establish to what degree the additional cost of using this technique would offset the benefit of doubled overall PE, set against food security and land use issues which may ultimately prove a critical socioeconomic factor. This additional cost is likely to be minimised for photobioreactor designs utilising optical fibre light-delivery (Erickson et al. 2011) which would require only small dichroic mirrors. The optical damage threshold of the mirrors is typically very high $\left(>1,000 \mathrm{MW} / \mathrm{m}^{2}\right.$; manufacturer's specification for the mirror used in this study), three orders of magnitude 
greater than the peak solar intensity at a concentration factor of 1,000 .

\section{Conclusion}

Dichroic beam-sharing offers a practical opportunity for increasing photosynthetic activity by up to $100 \%$. This study demonstrated a $71 \%$ increase in the combined photosynthetic activity of A. platensis and $R$. sphaeroides, which have been studied in the production of sustainable food and $\mathrm{H}_{2}$ fuel, respectively. Losses were associated mainly with $A$. platensis and attributed to the partial reflection of useful 'blue' (400-550 nm) light by the dichroic mirror. The demonstrated principle indicates that solar photosynthetic activity could be increased without requiring additional land or sunlight, using existing technology to enhance the sustainable production of foods, fuels and chemicals via duplexed photobioreactor systems.

Acknowledgments This study was funded by an MRC/ BBSRC/EPSRC Discipline Hopping Award (Photonic Solutions for Bioenergy; No. G0902337).

Open Access This article is distributed under the terms of the Creative Commons Attribution License which permits any use, distribution, and reproduction in any medium, provided the original author(s) and the source are credited.

\section{References}

Archer MD, Barber J (2004) Molecular to global photosynthesis. World Sci. ISBN: 978-1-86094-256-3

ASTM (American Society for Testing and Materials) (2010) E927-10: standard specification for solar simulation for terrestrial photovoltaic testing (Report). doi:10.1520/ E0927-10. http://www.astm.org/Standards/E927.htm

Blankenship RE, Madigan MT, Bauer CE (1995) Anoxygenic photosynthetic bacteria. Kluwer Academic, Dordrecht

Chen SL (1952) The action spectrum for the photochemical evolution of oxygen by isolated chloroplasts. Plant Physiol 27:35-48

Chen H-B, Wu J-Y, Wang C-F, Fu C-C, Shieh C-J, Chen C-I, Wang C-Y, Liu Y-C (2010) Modeling on chlorophyll a and phycocyanin production by Spirulina platensis under various light-emitting diodes. Biochem Eng J 53:52-56

Erickson D, Sinton D, Psaltis D (2011) Optofluidics for energy applications. Nat Photon 5:583-590

French CS (1937) The rate of $\mathrm{CO}_{2}$ assimilation by purple bacteria at various wave lengths of light. J Gen Physiol 21:7187

Guo C-L, Zhu X, Liao Q, Wang Y-Z, Chen R, Lee D-J (2011) Enhancement of photo-hydrogen production in a biofilm photobioreactor using optical fiber with additional rough surface. Bioresour Technol 102:8507-8513

Gurgel AC, Reilly JM, Paltsev S (2008) Potential land use implications of a global biofuels industry. MIT joint program on the science and policy of global change. Report no. 155. http://hdl.handle.net/1721.1/41521. Accessed 6 Sept 2010

Haxo FT, Blinks LR (1950) Photosynthetic action spectra of marine algae. J Gen Physiol 33(4):389-422

Kerr RA (2011) Peak oil production may already be here. Science 331:1510-1511

Metzger P, Largeau C (2005) Botryococcus braunii: a rich source for hydrocarbons and related ether lipids. Appl Microbiol Biotechnol 66:486-496

Miyamoto K, Ohta S, Nawa Y, Mori Y, Miura Y (1987) Hydrogen production by a mixed culture of a green alga Chlamydomonas reinhardtii and a photosynthetic bacterium Rhodospirillum rubrum. Agric Biol Chem 51:13241391

Nogi Y, Akiba T, Horikosji K (1985) Wavelength dependence of photoproduction of hydrogen by Rhodopseudomonas rutila. Agric Biol Chem 49:35-38

Orozco RL, Redwood MD, Reza A, Leeke G, Santos R, Macaskie LE (2012) Hydrothermal hydrolysis of starch with $\mathrm{CO}_{2}$ and detoxification of the hydrolysates with activated carbon for bio-hydrogen fermentation. Int J Hydrog Energy 37:6545-6553

Raoof B, Kaushik BD, Prasanna R (2006) Formulation of a lowcost medium for mass production of Spirulina. Biomass Bioenerg 30:537-542

Redwood MD, Orozco R, Majewski AJ, Macaskie LE (2012a) Electro-extractive fermentation for efficient biohydrogen production. Bioresour Technol 107:166-174

Redwood MD, Orozco R, Majewski AJ, Macaskie LE (2012b) An integrated biohydrogen refinery: synergy of photofermentation, extractive fermentation and hydrothermal hydrolysis of food wastes. Bioresour Technol 119:384-392

Work VH, D'Adamo S, Radakovits R, Jinkerson RE, Posewitz MC (2012) Improving photosynthesis and metabolic networks for the competitive production of phototrophderived biofuels. Curr Opin Biotechnol 23:290-297 